\title{
HACIA UNA ESPECIALIZACIÓN EN INTERPRETACIÓN EN EL ÁMBITO \\ DE LA VIOLENCIA DE GÉNERO: INVESTIGACIÓN, FORMACIÓN Y PROFESIONALIZACIÓN
}

\author{
Carmen Toledano Buendía \\ ctoledan@ull.edu.es \\ Universidad de La Laguna \\ María Isabel Abril Martí \\ miabril@ugr.es \\ Universidad de Granada \\ Maribel del Pozo Triviño \\ mdelpozo@uvigo.es \\ Universidad de Vigo \\ Laura Aguilera Ávila \\ laguiler@ull.edu.es \\ Universidad de La Laguna
}

\section{Resumen}

El creciente interés académico y científico que en los últimos años se ha prestado a la traducción/interpretación para los servicios públicos parece haber puesto de manifiesto de manera incuestionable la importancia que tiene la formación y la profesionalización de los/las intérpretes para poder asegurar una prestación de servicios de mediación lingüística con todas las garantías de calidad.

La interpretación en el ámbito de la violencia de género no es una excepción. Las peculiaridades que puede presentar la mediación lingüística con víctimas extranjeras de violencia de género en los diferentes contextos y fases de intervención parecen 
requerir una especialización adicional dentro de la interpretación para los servicios públicos.

En este trabajo buscamos aproximarnos de manera general a esas necesidades específicas de especialización, estudiar las experiencias existentes en este campo en otros países y esbozar las posibles actuaciones que podrían llevarse a cabo para alcanzarla desde un punto de vista investigador, formativo y profesional.

\begin{abstract}
"Towards the specialization of public service interpreters in the area of gender-based violence. Research, training and professionalization"

In the past few years the academic and scientific community has shown increased interest in the field of Public Service Translation and Interpreting. This has clearly highlighted the importance of training and professionalization to ensure the provision of top quality language mediation services.

Interpreting in the field of gender-based violence is no exception. Providing language mediation to foreign victims in different areas and stages of gender violence assistance services involves specific features which would seem to require further specialized training for the public service interpreters involved.

This paper intends to provide an overview of such specific needs for specialized training, analyze the experience of other countries in this area, and outline possible ways to achieve the necessary specialization from the standpoint of research, training and professional practice.
\end{abstract}

Palabras clave: Traducción/Interpretación en los servicios públicos. Mediación lingüística. Violencia de género. Formación. Profesionalización.

Keywords: Public Service Translation/Interpreting. Language mediation. Gender-based violence. Training. Professionalization.

Manuscript received on February 15, 2013 and accepted for publication on January 19, 2014. 


\section{Introducción}

A diferencia de países como Australia, EE.UU., Canadá, Suecia o Reino Unido, España no ofrece una respuesta integral a los problemas de comunicación que se plantean entre la población extranjera y los servicios públicos. Con excesiva frecuencia -casi cabría decir con normalidad- los problemas de intermediación lingüística que surgen en diferentes ámbitos de los servicios públicos (comisarías, escuelas, hospitales, ayuntamientos, juzgados, etc.), derivados del insuficiente conocimiento del castellano, o de las demás lenguas oficiales por parte de la población extranjera, se solucionan de manera improvisada, recurriendo a personas empleadas de otras áreas de la propia institución con ciertos conocimientos de idiomas, a voluntariado, a los y las propias acompañantes de las personas usuarias (a veces menores o familiares implicados en el conflicto en el que se trata de mediar), etc. Así lo demuestran distintas publicaciones que analizan el panorama español, entre las que cabe destacar Ortega Herráez (2010); Ortega, Abril \& Martin (2009); Del Pozo \& Gómez (2012) y Raga \& Valero (2006). Asimismo, las investigaciones centradas en el análisis de las prestaciones de mediación lingüística realizadas por intérpretes no profesionales han demostrado inequívocamente las importantes deficiencias en los procesos de comunicación que se derivan de estos hábitos (Pöllabauer 2008, Schweda-Nicholson 1989, Pöchhacker 2000, Pöchhacker \& Kadric 1999, Sánchez-Reyes \& Martín Casado 2005).

Estas deficiencias dificultan o incluso impiden el acceso y el disfrute igualitario de los servicios públicos, pueden poner en peligro el bienestar además de la salud de la persona usuaria de los mismos, y redundan en el mal aprovechamiento de los recursos que las instituciones ponen al servicio de la ciudadanía (Abraham \& Fiola 2006; Abril \& Martin 2011; Bischoff 2003; Bowen 2010; Cambridge 1999; De Luna 2010; Díaz 2009, Flores et al. 2003; Jacobs et al. 2003; Kelly \& Bancroft 2007; Ortega Herráez 2010; Sánchez-Reyes \& Martín Casado 2005).

Esta problemática, constatable de forma genérica en todos los ámbitos de actuación de la disciplina de la interpretación para los servicios públicos, se agrava en el caso específico de la intermediación en casos de violencia de 
género donde las consecuencias de cuestiones como la imprecisión o la falta de neutralidad o de confidencialidad en la actuación del/la intérprete puede tener consecuencias fatales (Polzin 2007: 23).

Si bien somos conscientes de que es necesario llevar a cabo más investigación en este ámbito para poder sentar las bases de una propuesta curricular sólida, consideramos que la interpretación para víctimas de violencia de género requiere una formación específica, ya que entran en juego numerosos aspectos que no se tratan en cursos más generales de interpretación para los servicios públicos. Entre dichos aspectos cabe mencionar, a modo de ejemplo: derechos de las víctimas y legislación sobre violencia de género, ciclo de la violencia, gestión del estrés emocional, etc. (Hale 2011).

\section{Mujeres extranjeras, violencia de género y barreras lingüísticas}

Las mujeres inmigrantes constituyen un colectivo con una problemática específica, ya que aúnan dos condiciones que promueven la discriminación: la cuestión de género y la de procedencia. Esto las coloca en una posición de mayor precariedad con respecto a las mujeres nacionales, por un lado, y a los hombres inmigrantes por otro, contando con menos posibilidades de mejorar su situación, y menores oportunidades de educación y de participación. Esta posición de desventaja las conduce a una situación de mayor vulnerabilidad y a una particular desprotección en los casos de violencia doméstica (Amnistía Internacional 2007). La Macroencuesta sobre Violencia de Género de 2011, realizada por el Centro de Investigaciones Sociológicas (CIS) en colaboración con el Gobierno, alerta de que "la prevalencia de la violencia de género entre las mujeres extranjeras duplica la de las mujeres españolas". Según el citado documento, el 10,1\% de las mujeres españolas sufrieron violencia de género alguna vez en la vida y el 2,8\% en algún momento durante el año 2010, frente al $20,9 \%$ y $5,8 \%$, respectivamente, de las mujeres extranjeras.

El porcentaje de víctimas extranjeras de este tipo de violencia no es nada despreciable. Según el informe sobre víctimas mortales por violencia de genero elaborado por el Consejo General del Poder Judicial, el número de mujeres muertas por sus parejas o ex parejas a lo largo de 2011 ascendió a 62, de las cuales $40(65 \%)$ eran españolas y 22 (35\%) eran extranjeras, porcentaje que adquiere mayor relevancia si se tiene en cuenta que las mujeres extranjeras representaban el 11,5\% del total de la población femenina en España (2011: 15-17). El 74\% de las fallecidas no había denunciado previamente malos tratos.

Si analizamos la nacionalidad de las denunciantes, en el primer trimestre de 2013 el 67\% de las denunciantes eran de nacionalidad española frente al 
$33 \%$ de denunciantes extranjeras, porcentajes similares a los de trimestres anteriores. También resulta interesante observar cómo existen diferencias en el porcentaje de mujeres que acaban renunciando al proceso (con independencia de que exista el deber de investigación por parte de las autoridades). En términos globales en un $12,01 \%$ de los casos se renuncia al proceso y de ellos un 59\% corresponde a españolas frente al $41 \%$ de extranjeras. ${ }^{1}$

Las mujeres extranjeras víctimas de violencia de género se encuentran en una situación de especial vulnerabilidad y desprotección como consecuencia de factores tan diversos como una precaria situación laboral y administrativa, el contexto de desarraigo familiar y social, el escaso conocimiento de sus derechos y la dificultad para acceder a información sobre los mismos, aspectos que, unidos al miedo al que se ven sometidas, les hace muy difícil escapar del círculo de violencia del que son víctimas. A estos factores debemos también añadir, en muchos casos, el desconocimiento de la lengua de la comunidad de acogida como obstáculo adicional que agrava su sentimiento de aislamiento y desprotección y que puede llegar a actuar como un elemento disuasorio a la hora de solicitar ayuda y denunciar: "language access plays a central role in the ability of survivors to progress in their journeys to safety" (Huelgo et al. 2006: 5).

Además, las barreras idiomáticas también limitan la capacidad de las administraciones de acercarse e informar a las posibles usuarias y dificultan su atención y asistencia. Así lo indican los resultados del cuestionario llevado a cabo en la Red de Intervención Especializada en Violencia de Género, en la isla de Tenerife, sobre la evaluación de la mediación lingüística, y realizado por la Unidad Orgánica de Violencia de Género del Cabildo de Tenerife (2011). Según las profesionales encuestadas, junto a las dificultades que el idioma supone en la prestación de los diferentes servicios en las distintas áreas de intervención (información, asesoramiento, valoración e intervención psicosocial de la víctima, asesoramiento jurídico, etc.), también se suman los problemas que encuentran para mostrar a las usuarias la utilidad del servicio, la importancia de que continúen acudiendo al mismo ("engancharla" al servicio), y los riesgos que conlleva volver con el agresor. Asimismo, las profesionales encuestadas aseguran que contar con servicios de asistencia lingüística

1. Estadísticas publicadas por el Observatorio contra la violencia doméstica y de género y el Consejo General del Poder Judicial. Disponible en http://www.observatorioviolencia. org/upload_images/File/DOC1373452998_4T\%202012-Violencia\%20sobre\%20la\%20 Mujer.pdf. Estas estadísticas no hacen un desglose pormenorizado por país de procedencia, por lo que no es posible determinar cuáles serían potenciales usuarias de servicios de interpretación de entre los datos correspondientes a mujeres extranjeras. 
de calidad animaría a otras víctimas a denunciar su situación (Toledano \& Fernández 2012).

\section{Derechos lingüísticos de las víctimas e instrumentos para su cumplimiento a nivel internacional}

La importancia de la mediación lingüística en la atención a las mujeres extranjeras víctimas de violencia de género que no dominan la lengua de la comunidad en la que se encuentran ha pasado inadvertida a las administraciones y los gobiernos de muchos países y entre ellos España. Prueba de ello, por ejemplo, es el hecho de que la Ley Orgánica 1/2004, de 28 de diciembre de Medidas de Protección Integral contra la Violencia de Género (BOE, 2004) ni siquiera incluye el derecho de las inmigrantes a servicios lingüísticos al no considerar una dificultad "para acceder a los servicios previstos en esta Ley" el desconocimiento del idioma. Partiendo de esta laguna, en la práctica es habitual encontrar soluciones no profesionales (Molina Gutiérrez 2006).

No obstante, dentro de la Unión Europea se trata de una de las líneas de actuación prioritarias a través de su programa de Justicia Penal, y más concretamente, del programa Daphne III. Además, no se puede pasar por alto la Directiva 2012/29/UE del Parlamento Europeo y del Consejo de 25 de octubre de 2012 por la que se establecen normas mínimas sobre los derechos, el apoyo y la protección de las víctimas de delitos, y por la que se sustituye la Decisión marco 2001/220/JAI del Consejo (DOUE, 2012), en la que se presta especial atención, entre otras, a las víctimas de violencia de género y al derecho de estas a oír y a ser oídas, así como a su acceso a los servicios de apoyo y de traducción e interpretación gratuitos durante las distintas fases del proceso penal. Otra de las particularidades de esta nueva regulación es que se otorga la consideración de víctima a los familiares de las personas fallecidas como consecuencia de una infracción penal, aspecto de especial relevancia en el caso de la violencia de género en nuestro país.

Fuera de las fronteras europeas también existe un interés creciente por las particularidades de la intermediación lingüístico-cultural en la atención a la violencia de género y no faltan ejemplos de creación de recursos didácticos, como los del National Center for State Courts (Access to Justice: Limited English Proficiency LEP y Access to Protection Orders), ${ }^{2}$ o el Department of Justice Office on Violence Against Women (Resource Guide for Advocates \&

2. http://www.ncsc.org/microsites/access-to-justice/home/Topics/LEP-Self-RepresentedLitigants.aspx 
Attorneys on Interpretation Services for Domestic Violence Victims), ${ }^{3}$ ambos en EE.UU., o los materiales editados por la Community Relations Commission for a Multicultural New South Wales (Using Interpreters in Domestic Violence and Sexual Assault Matters) ${ }^{4}$ de Australia, por citar solo algunos.

En este último país se desarrolló entre octubre de 2010 y noviembre de 2011 un amplio proyecto titulado "Breaking through the language barrier: Empowering refugee and immigrant women to combat domestic and family violence through cultural and language training". Fue liderado por la doctora Sandra Hale, una de las investigadoras más reputadas internacionalmente en el ámbito de la interpretación en los servicios públicos, y contó con financiación gubernamental a través de las autoridades de Nueva Gales del Sur (NSW Department of Premier and Cabinet, Domestic and Family Violence Grant Scheme). Este proyecto surgió como respuesta a un problema habitual en las sociedades receptoras de inmigración: la falta de intérpretes profesionales para comunidades cuyas lenguas no están suficientemente representadas en los servicios lingüísticos existentes, lo que significa que las necesidades de comunicación están siendo cubiertas por no profesionales, a menudo miembros de dichas comunidades, por ser las únicas personas disponibles. Este proyecto partió de un profundo análisis de necesidades y recursos existentes, para plantear a continuación un programa de formación cultural y lingüística con un enfoque doble: por un lado, una formación mínima (un día) en cuestiones relativas a derechos de la mujer y violencia de género; por otro lado, un programa de formación de 40 horas especializado en la interpretación en contextos de violencia doméstica (Hale 2011).

También es digno de destacar el proyecto piloto para la provisión de intérpretes especializados que se desarrolló a finales de la década de 1990 en dos Juzgados de Violencia Doméstica y en el Women's College Hospital de la ciudad de Toronto (Abraham y Oda 2000). Este proyecto se complementaría poco después con la formación ampliada de los intérpretes para los programas de trabajo con los maltratadores, que por mandato judicial debían participar en un programa de educación y atención psicológica (Oda y Joyette 2003). En la actualidad, la provincia de Ontario lleva a cabo un programa denominado LIS (Language Interpreters Service) cuyo objetivo es proporcionar intérpretes a los servicios públicos para que puedan comunicarse con las usuarias con conocimientos limitados de inglés o francés que sean víctimas de violencia

3. http://www.dcf.state.fl.us/programs/domesticviolence/dvresources/docs/Interpretation ResourceGuide.pdf

4. http://www.crc.nsw.gov.au/publications/documents/interpreters_domestic_violence 
de género, violencia sexual o trata. Este servicio también está disponible para personas con limitaciones auditivas o sordas. El servicio de interpretación corre a cargo del gobierno de la provincia y está disponible para los profesionales de servicios como policía, sanitarios, judicial, etc. mediante agencias que en su mayoría son organizaciones sin ánimo de lucro. Dichas agencias adquieren el compromiso de formar a los/as intérpretes siguiendo un programa diseñado por el propio gobierno.

\section{Justificación de la necesidad de formación específica para los/as profesionales de la interpretación}

Como ya se ha señalado en párrafos anteriores, la interpretación en los contextos vinculados a la intervención con víctimas de violencia de género es una práctica compleja que vincula elementos materiales, humanos e intelectuales provenientes de muy variados ámbitos del conocimiento y la praxis -con dimensiones, al menos, sociológicas, psicológicas, médicas, antropológicas, jurídicas y lingüísticas-, que deben, además, coordinarse de manera equilibrada para proporcionar una atención integral a la víctima. La presencia del/a intérprete puede requerirse en diferentes fases además de en distintos servicios y recursos y para encuentros con fines, expectativas, necesidades y dinámicas comunicativas diferentes. Este hecho complica su labor y requiere la definición de un perfil profesional especializado así como la creación de unos protocolos de actuación.

Aspectos que ya de por sí son controvertidos y que plantean dilemas a los/ as intérpretes en los servicios públicos se magnifican en casos de violencia de género y pueden derivar en el fracaso de la asistencia a la víctima. Durante el desarrollo de los proyectos de Canadá y Australia descritos anteriormente se identificaron una serie de deficiencias en la intervención de intérpretes no profesionales, así como expectativas de los/as agentes entrevistados, que se integraron en los programas de formación como destrezas específicas para la mediación lingüística y cultural en los contextos de violencia de género (cf. Abraham \& Oda 2000, Hale 2011). Por ejemplo, la capacidad para verbalizar vocabulario obsceno $\mathrm{u}$ ofensivo al interpretar el relato de víctimas y agresores. Cabe explicar que, en determinadas culturas, el que una mujer exprese este tipo de vocabulario, aunque sea como reproducción de un relato ajeno, es socialmente inaceptable y puede afectar a su reputación, especialmente si pertenece a una comunidad pequeña.

De forma similar, el/la intérprete ha de tener unos conocimientos profundos de cuestiones específicas como el concepto de violencia de género, la forma de afrontar la violencia, el papel de los géneros en sociedades dominadas por 
el hombre, etc.; en definitiva, cuestiones socioculturales que pueden condicionar las estrategias comunicativas, el sentido de la relevancia o la propiedad en la transmisión de la información, las formas de expresión...o la actitud de los interlocutores. Por último, cuestiones éticas como la confidencialidad o la imparcialidad se señalaron especialmente en estos programas como insoslayables. La violación de la confidencialidad puede afectar a la seguridad personal de la víctima, y la sobreintervención del/la intérprete que aconseja a la víctima que solucione los problemas en casa o que la juzga como culpable no hace sino aumentar su desprotección y desamparo.

Por otra parte, la mediación lingüística en estos contextos requiere la presencia de intérpretes profesionales que cuenten no solo con formación en traducción e interpretación para los servicios públicos en general, sino también con una especialización adicional que los capacite para trabajar en la variada serie de encuentros que van a tener lugar a lo largo del proceso de intervención y que permita su integración en el equipo de especialistas que se ocupa de esta problemática social.

Sobre la base de este perfil específico, el proyecto para los juzgados de violencia doméstica de Toronto anteriormente mencionado incluyó entre las competencias objetivo de la formación el conocimiento de los diferentes programas de intervención, los protocolos de actuación, el funcionamiento de los servicios y el papel de los/as agentes, así como los documentos habitualmente utilizados. La formación en aspectos teóricos sobre género, sensibilización con la situación de las víctimas y la empatía se integraron también entre los contenidos desarrollados (Abraham \& Oda 2000).

Por su parte, el programa de Nueva Gales del Sur incluyó contenidos teóricos sobre el papel de la mujer en Australia, los derechos de la mujer y el marco jurídico internacional que la protege contra la violencia, el concepto de violencia de género y el funcionamiento del sistema australiano de asistencia a víctimas de violencia doméstica. El curso de interpretación propiamente dicha, consistió en técnicas de interpretación, ética y conocimientos de los ámbitos de servicios públicos más directamente relacionados con la asistencia a víctimas, es decir, servicios sanitarios y jurídicos.

Por último, la mediación lingüística en muchas ocasiones se requiere en encuentros con gran carga emocional, con personas que han sido traumatizadas, lo que añade complejidad a la comunicación y al proceso de interpretación. Relacionado con este aspecto emocional, Huelgo et al. (2006: 6) incluyen entre las recomendaciones para una capacitación profesional, el entrenamiento y el apoyo a estos y estas profesionales para trabajar en situaciones 
muy delicadas y para poder interpretar testimonios y experiencias verdaderamente traumáticas:

It is not only survivors who are traumatized by their experiences. Court interpreters who hear and relay the horrific experiences of survivors of domestic violence, sexual assault or child abuse may experience vicarious or secondary trauma. Without adequate training and supervisory support, this work can take too heavy a toll, to the point of impeding an interpreter's ability to keep performing such an essential job. Therefore, court interpreters need comprehensive training in sensitivity to these difficult issues as well as a clear supervisory channel for professional development and support.

Recordemos que la especialización de los/as profesionales y agentes vinculado/as a la atención a víctimas de violencia de género en todos sus ámbitos se considera esencial. De hecho, así lo ha reconocido expresamente el Grupo de Expertos y Expertas en Violencia Doméstica y de Género del Consejo General del Poder Judicial en su informe del 2011 sobre los problemas de aplicación de la Ley Orgánica 1/2004:

Se entiende igualmente que la formación de los Abogados en esta materia debiera ser específica, homogénea y continua, debiendo considerarse este tipo de formación tan obligatoria para los letrados como para el resto de profesionales que intervienen en procesos de esta naturaleza, debiendo comprender además las especificidades derivadas de los supuestos de discapacidad y de exclusión social, que, en ocasiones afectan a las víctimas (mujeres extranjeras en situación administrativa irregular, mujeres vinculadas a la explotación sexual o que sufren otro tipo de exclusión social...) (2011: 28).

Sin embargo, en nuestro país esta recomendación no solo es totalmente ignorada en el caso de los/as intérpretes participantes en estos procesos, sino que las soluciones a las que se recurre para salvar las barreras lingüísticas se suelen improvisar echando mano de personas sin cualificación, e incluso de familiares de las propias víctimas.

\section{Líneas de actuación desde el punto de vista investigador, formativo y profesional}

Ante esta situación de desprofesionalización y, lo que es más grave aún, de negligencia y desatención a este vital eslabón en la cadena de atención e intervención con las víctimas extranjeras que es la provisión de asistencia lingüística, nos preguntamos cuáles son las posibles medidas que desde el ámbito académico podemos empezar a llevar a cabo para cambiarla.

En situaciones de desprofesionalización como la que caracteriza la prestación de servicios de traducción e interpretación en los servicios públicos en nuestro país, las universidades pueden desempeñar una labor muy importante 
de educación y "normalización" de la disciplina y de la práctica de la interpretación. Esta normalización...

not in the sense of making what happens in it normal but rather subjecting it to norms, will guarantee professionalism and in return this professionalism will guarantee the very existence of the discipline. [...] Education provides knowledge and skills; it also deepens understanding, reinforces standards and leads to consensus. Universities can and should take on the responsibility of coalescing all these activities. They relate as much to reflective practice and self criticism as to raising the profile of trained professionals. Both these factors are essential to achieving the level of professionalism in which norms are rooted (Toledano Buendía 2010).

Consideramos que desde la universidad se puede trabajar en torno a tres ámbitos de actuación: investigación, formación y práctica profesional. Si bien se trata de tres líneas de trabajo distintas, éstas deben estar interrelacionadas. Lamentablemente, como señala Hale (2007: 197), esta relación en el campo de la traducción y la interpretación en los servicios públicos es todavía insuficiente, ya que la transferencia de los resultados de la investigación a la práctica profesional sigue siendo en muchos casos una asignatura pendiente. Las relaciones entre la investigación, la formación y la práctica profesional son bidireccionalmente necesarias con el fin de alcanzar la eficacia del servicio, el reconocimiento y profesionalización de la actividad, la calidad de su enseñanza y la consolidación disciplinar de su campo de investigación.

\subsection{Líneas de actuación en investigación}

En el ámbito de la investigación, el estudio de la traducción y la interpretación en los servicios públicos aplicada al contexto de la violencia de género es un campo prácticamente inexplorado. Hasta el momento no hemos podido constatar la existencia de investigaciones que aborden de forma específica la práctica de la interpretación y de la traducción en la atención integral a las víctimas de la violencia de género a excepción de las realizadas para el proyecto piloto de Ontario y el programa de Nueva Gales del Sur mencionados anteriormente, y de algunas recomendaciones prácticas publicadas en Newsletters de asociaciones profesionales (Huelgo et al. 2006; Polzin 2007).

Si bien la formación es imprescindible en todo proceso de profesionalización y especialización, consideramos que la investigación es un paso previo fundamental sobre el cual esta se debe asentar. Los esfuerzos en materia formativa deben estar basados en una investigación sistemática previa del contexto sociolaboral y de la realidad epistemológica que nos permita conocer en profundidad los diferentes campos de actuación, los tipos de encuentros y 
agentes, sus necesidades y expectativas, con el fin de identificar las especificidades de las interacciones comunicativas interlingüísticas e interculturales.

En el caso que aquí nos ocupa parece evidente la pertinencia, en primer lugar, de contar con un mapa de las necesidades de prestación de servicios de interpretación en el ámbito de la violencia de género -hoy inexistente o, en el mejor de los casos, parcial-y de sus posibles soluciones. Para ello es necesario conocer y analizar la demanda de personal de traducción e interpretación para los servicios públicos en el ámbito de la violencia de género, usuario/as de los servicios y frecuencia de su uso, los idiomas de trabajo, necesidades a las que responde, cómo se cubren en la actualidad y deficiencias detectadas por agentes y usuarias. Posteriormente, una segunda línea de investigación estaría orientada a la definición y análisis de los contextos de trabajo, las dinámicas sociales, culturales y lingüísticas que en ellos acontecen, las expectativas de los/as agentes o profesionales de los servicios, y los principales problemas de comunicación interlingüística e intercultural que se plantean.

Estos objetivos forman parte de las líneas de actuación que, desde el proyecto Speak Out for Support (SOS-VICS) (JUST/2011/JPEN/2912), liderado por la Universidade de Vigo, ${ }^{5}$ y con financiación de la Unión Europea a través de su programa de Justicia Penal, se pretenden abordar al objeto de cubrir las lagunas detectadas en lo que a la interpretación en el ámbito de la violencia de género se refiere. En una primera fase del proyecto se ha llevado a cabo la compilación y análisis de las necesidades de comunicación de todas las personas implicadas: víctimas, diferentes agentes e intérpretes (cf. Del Pozo, Vaamonde et al., en prensa-a y en prensa-b). Tras el análisis estadístico de los datos obtenidos en esta primera fase se han establecido patrones que han ayudado a crear los recursos contemplados en la segunda fase, durante la cual se han desarrollado diferentes materiales para todas las partes implicadas: víctimas, agentes e intérpretes. Entre estos se incluyen: una página web de información general ${ }^{6}$, información multilingüe para las víctimas en formato de vídeo y folleto, una guía de buenas prácticas para agentes (cf. Borja y Del Pozo, 2014), así como material y recursos especializados para

5. En el proyecto participan investigadores/as de las universidades de Vigo, La Laguna, Alcalá, Barcelona, Vic, Jaume I, Granada, Alicante y Salamanca, a los que se unen experto/as externos/as como, entre otros, el Dr. Erik Hertog de la Universidad Católica de Lovaina-Lessius, la Asociación Profesional de Traductores e Intérpretes Judiciales y Jurados (APTIJ), el Instituto de Medicina Legal de Galicia (IMELGA), la Fundación Academia Europea Yuste, la Rede de Mulleres contra os Malos Tratos de Vigo, la Fundación Ana Bella, la Unidad Orgánica de Violencia de Género del Cabildo de Tenerife y la Asociación Mercedes Machado, así como representantes de la judicatura y la fiscalía.

6. http://cuautla.uvigo.es/sos-vics/ 
intérpretes y para profesionales que se ocupan de su formación en forma de plataforma web (Abril, 2014) y un libro de formación para intérpretes (cf. Toledano y Del Pozo, 2014). La tercera fase del proyecto está dedicada a la divulgación de la metodología y de los resultados. Este proyecto está desarrollando métodos innovadores de gran utilidad social para la protección de las víctimas que podrán transferirse a otros países de la Unión Europea, bien mediante la extrapolación de las experiencias y de las lecciones aprendidas, o bien mediante futuros proyectos transfronterizos con países que compartan características similares a las de España en el ámbito de la violencia de género. Conviene destacar que la atención a las víctimas y personas vulnerables es una de las prioridades de los programas de acción de la Unión Europea, de ahí el interés mostrado por el citado proyecto.

La transferencia de los resultados de las diferentes investigaciones a la sociedad, así como la propia colaboración interdisciplinar con investigadores/ as de otras áreas y profesionales de los diferentes ámbitos de intervención con víctimas de violencia de género en el desarrollo de las investigaciones, genera ya canales de comunicación y divulgación de la labor del/la intérprete, de su relevancia en el tratamiento a las víctimas y de las necesidades de profesionalización, aspectos que con mucha frecuencia son totalmente desconocidos. En esta línea el proyecto SOS-VICS representa un modelo no solo por la composición del grupo de investigación multidisciplinar integrado por investigadores de disciplinas como la traducción e interpretación, la psicología, el trabajo social, el derecho, la sociología, la medicina, la informática o la estadística, sino por la constante colaboración e interacción con profesionales a través de talleres y mesas de trabajos. Esta metodología ha supuesto un enriquecimiento mutuo, que sin duda redundará en la mejora de la atención integral a las víctimas.

\subsection{Líneas de actuación en formación}

Como apuntábamos en páginas anteriores, la formación es un paso esencial en la especialización y profesionalización de los/as intérpretes para asegurar una prestación de servicios de calidad. El diseño de los programas de formación para intérpretes especializados/as en el ámbito de la violencia de género deberá basarse en los resultados de los estudios previos sobre las necesidades de interpretación y las características de los contextos de trabajo. Éstos nos permitirán definir el perfil humano y profesional del/a intérprete y su labor, así como identificar las competencias que debe desarrollar y los conocimientos que debe adquirir. Asimismo, el estudio de las expectativas de quienes participan en los procesos y las necesidades de cada ámbito de intervención 
permitirá definir el modus operandi del o de la intérprete, delimitar su función y modelos de actuación y los grados pertinentes de visibilidad según el contexto y las expectativas. Aspectos relacionados con las lenguas de trabajo, el tipo de alumnado, su formación y experiencia previa, etc. son elementos adicionales que deben tenerse en cuenta en el diseño del programa de formación (duración, naturaleza reglada o no reglada, etc.). En este sentido resulta fundamental la colaboración y el trabajo de las asociaciones de traductores/ intérpretes profesionales como canal de formación permanente y especialización, sobre todo considerando que estas asociaciones cuentan con una mayor flexibilidad en el diseño de su oferta formativa y en la admisión de participantes hablantes de lenguas minoritarias que las instituciones académicas propiamente dichas. En el proyecto SOS-VICS esta información ha sido obtenida a través del análisis de los resultados de diferentes trabajos de campo realizados a partir de encuestas, entrevistas y cuestionarios a todos los participantes en los encuentros con víctimas extranjeras de violencia de género: agentes, víctimas e intérpretes.

\subsection{Lineas de actuación en profesionalización}

Finalmente, y con una orientación aplicada, la tercera línea de actuación iría dirigida a una mejora de la práctica profesional a través del desarrollo de diversos recursos de apoyo a la mediación en encuentros interlingüísticos e interculturales con víctimas y supervivientes de violencia de género. La aplicación de los resultados de diferentes estudios e investigaciones teóricodescriptivos puede orientarse a la elaboración de materiales como glosarios, recursos bibliográficos, FAQ, recomendaciones de actuación, recopilación de documentos y traducciones, guías de buenas prácticas para agentes, etc. que suponen un apoyo directo a la práctica de la interpretación y a la labor de los/ as diferentes profesionales implicados/as en la mediación e intervención con víctimas y supervivientes que no hablan castellano o las demás lenguas oficiales. Este material generado puede resultar de utilidad no solo a intérpretes y asociaciones profesionales de intérpretes sino a entidades públicas y privadas, ONG, asociaciones de víctimas y supervivientes y cualquier organismo técnico con competencias en materia de violencia de género.

En el ámbito de la traducción especializada encontramos un ejemplo de este tipo de material, orientado a mejorar la práctica profesional de los traductores en el proyecto JUDGENTT, del grupo de investigación de Géneros 
Textuales para la Traducción (GENTT) de la Universitat Jaume I. ${ }^{7}$ De manera específica para la interpretación con víctimas de violencia de género, destacamos los materiales y recursos desarrollados en el proyecto SOS-VICS mencionados anteriormente.

\section{Conclusiones}

Cuando las mujeres víctimas de violencia de género deciden poner fin a la misma tienen que enfrentarse a múltiples situaciones complicadas y pasar por un trance vital en el que pueden ver cuestionada su seguridad e integridad (dificultad para entender y asumir su condición, recorrido por diferentes servicios y procesos, dificultad para acceder a información de calidad, etc.). En el caso de las mujeres extranjeras su conflicto es doble, ya que deben, además, superar la barrera del idioma. Asimismo, la ausencia de intérpretes profesionales en el proceso de atención a las mujeres extranjeras que no entienden el castellano ni las demás lenguas oficiales en España, hace que, en ocasiones, los recursos que las administraciones públicas ponen al servicio de las víctimas no lleguen a ellas o, si llegan, no produzcan los resultados esperados.

Por esta razón podemos concluir que la incorporación de personal de traducción e interpretación especializado en el ámbito de la violencia de género no es solamente un requisito indispensable para la correcta atención a las mujeres que la sufren, sino que su ausencia en el proceso supone una vulneración de los derechos de las víctimas y también un desaprovechamiento de los recursos que el Estado pone a su disposición.

No obstante, y dado que la interpretación en los servicios públicos en general y la interpretación en el ámbito de la violencia de género en particular son disciplinas poco estudiadas y profesiones con poca trayectoria, es preciso llevar a cabo investigación ligada tanto a la formación de intérpretes como a la práctica profesional que tenga como resultado un servicio de calidad. Dicho servicio de calidad, prestado por profesionales con formación específica, reduciría el riesgo de revictimización de la mujer extranjera víctima de violencia de género, mejoraría la atención a la misma y redundaría en un mayor aprovechamiento de los recursos públicos.

7. http://www.gentt.uji.es/. Este grupo trabaja desde el año 2000 en la creación de una enciclopedia electrónica multilingüe de géneros textuales de los ámbitos jurídico, técnico y médico, que permita llevar a cabo investigaciones y que se plantee como una herramienta de consulta textual, conceptual, terminológica y lingüística para especialistas y no especialistas. 
Para concluir, consideramos que, dado que la violencia de género es un problema social que requiere una respuesta integral, la universidad, junto con las demás instituciones públicas, deben volcarse en buscar soluciones que contribuyan a la mejora de las condiciones de las mujeres víctimas de la violencia de género, también de las extranjeras que no entienden o no hablan las lenguas oficiales del estado. Por consiguiente, creemos que la universidad debe desempeñar un papel decisivo a la hora de investigar y formar en un ámbito, como es el de la interpretación para víctimas de violencia de género, que tiene aún mucho camino por recorrer para lograr el nivel de profesionalización requerido.

\section{Referencias bibliográficas}

ABRAHAM, Diana \& Marco Fiola. (2006) "Making the case for community interpreting in health care: from needs assessment to risk management." En: Hertog, Erik \& Bart Van der Veer (eds.) 2006. Taking Stock: Research and Methodology in Community Interpreting. Linguistica Antverpiensia 5, pp. 189-202.

ABRAHAM, Diana \& Melanie Oda. (2000) "The Cultural/Community Interpreter in the Domestic Violence Court: A Pilot Project." En: Abraham, Diana; Roda Roberts; Silvana Carr \& Aideen Dufour (eds.) 2000. The Critical Link 2; Interpreters in the Community. Amsterdam \& Philadelphia: John Benjamins, pp. 165-189.

ABRIL, Isabel \& Anne Martin. (2011) "La barrera de la comunicación como obstáculo en el acceso a la salud de los inmigrantes." En: Fernández, José Antonio \& María Nieves Moreno (eds.) 2011. Inmigración y crisis económica. Retos políticos y de ordenación jurídica. Granada: Comares, pp. 835-854.

ABRIL, Isabel (ed.) (2014). Web de formación SOS-VICS. Versión electrónica: $<$ http://cuautla.uvigo.es/sosvics-interpretes>

Amnistía Internacional. (2007) Más riesgos y menos protección. Mujeres inmigrantes en España frente a la violencia de género. Versión electrónica: <https://www.doc.es.amnesty.org/cgi-bin/ai/BRSCGI/MÁS\%20RIESGOS\%20 Y\%20MENOS\%20PROTECCIÓN\%20MUJERES\%20INMIGRANTES\%20 EN\%20ESPAÑA\%20FRENTE\%20A\%20LA\%20VIOLENCIA\%20DE\%20 GÉNERO?CMD=VEROBJ\&MLKOB=27230880101>

BISCHOFF, Alexander. (2003) Caring for migrant and minority patients in European hospitals. A review of effective interventions. Neuchâtel: Swiss Forum for Migration and Population Studies. Versión electrónica: <http://www.mfh-eu. net/public/files/mfh_literature_review.pdf>

BOE núm. 313, de 29 de diciembre de 2004. Ley Orgánica 1/2004, de 28 de diciembre, de Medidas de Protección Integral contra la Violencia de Género. Versión electrónica: <http://www.boe.es/buscar/doc.php?id=BOE-A-2004-21760> 
BORJA Albi, Anabel \& Maribel del Pozo (eds.) (2014) Orientaciones para trabajar con intérpretes en casos de violencia de género. Guía de buenas prácticas. Valencia: Tirant Lo Blanch.

Bowen, Sarah. (2010) 'From 'multicultural health' to 'knowledge translation'. Rethinking strategies to promote language access within a risk management framework." Jostrans 14, pp. 145-164.

CENTRO DE INVESTIGACIONES SOCIOlÓGICAS. (2011) Macroencuesta sobre Violencia de Género. Versión electrónica: <http://www.observatorioviolencia.org/ upload_images/File/DOC1329745747_macroencuesta2011_principales_ resultados-1.pdf>

CAMBridge, Jan. (1999) "Information loss in bilingual medical interviews through an untrained interpreter." Dialogue Interpreting. The Translator Studies in Intercultural Communication 5:2, pp. 201-219.

CONSEJO GENERAl Del PODER Judicial. (2011) Informe sobre víctimas mortales de la violencia de género y de la violencia doméstica en el ámbito de la pareja o ex pareja. Versión electrónica: <http://www.poderjudicial.es/cgpj/es/Temas/ Violencia_domestica_y_de_genero/Actividad_del_Observatorio/Informes_ de_violencia_domestica/Informe_sobre_victimas_mortales_de_la_violencia_ de_genero_y_de_la_violencia_domestica_en_el_ambito_de_la_pareja_o_ex_ pareja_en_2011>

Consejo General del Poder Judicial. Grupo de Expertos en Violencia Doméstica y de Género. (2011) Informe del Grupo de Expertos y Expertas en Violencia Doméstica y de Género del Consejo General del Poder Judicial acerca de los problemas técnicos detectados en la aplicación de la Ley Orgánica 1/2004. Versión electrónica: <http://www.poderjudicial.es/cgpj/es/Temas/Violencia_domestica_y_ de_genero/Grupos_de_expertos/Informe_del_Grupo_de_Expertos_y_ Expertas_en_Violencia_Domestica_y_de_Genero_del_Consejo_General_ del_Poder_Judicial_acerca_de_los_problemas_tecnicos_detectados_ en_la_aplicacion_de_la_Ley_Organica_1_2004>

DE LunA Jiménez de Parga, Pilar. (2010) "El intérprete judicial: ese interlocutor emocional entre el acusado y el juez." Diario La Ley 7368. Versión electrónica: <http://www.juecesdemocracia.es/congresos/xxvcongreso/ponencias/ El\%20interprete\%20Judicial.\%20Pilar\%20Luna.pdf>

Del Pozo, Maribel \& Elisa Gómez (eds.) (2012) Tradución e interpretación nos servicios públicos e asistenciais de Galicia. Primeiros pasos en investigación. Vigo: Universidad de Vigo.

Del Pozo Triviño, Maribel; Antonio Vaamonde Liste; David Casado-Neira; Silvia Pérez Freire; Alba Vaamonde Paniagua; Doris Fernandes del Pozo \& Rut Guinarte Mencía (en prensa-a). Informe general de la encuesta a agentes. Proyecto Speak Out for Support (SOS-VICS). 
Del Pozo Triviño, Maribel; Antonio Vaamonde Liste; David Casado-Neira; Silvia Pérez Freire; Alba Vaamonde Paniagua; Doris Fernandes del Pozo \& Rut Guinarte Mencía (en prensa-b). Informe de la encuesta Delphi a intérpretes. Proyecto Speak Out for Support (SOS-VICS).

DíAz Olalla, José Manuel. (2009) "Situación actual de la inmigración en España.

Desigualdades en salud." En: Morera Montes, Joaquín; Alberto Alonso Babarro \& Helena Huerga Aramburu (eds.) 2009. Manual de atención al inmigrante. Madrid \& Barcelona: Ergon, pp. 11-30.

Diario Oficial de la Unión Europea (de 14 de noviembre de 2012). Directiva 2012/29/UE del Parlamento Europeo y del Consejo de 25 de octubre de 2012 por la que se establecen normas minimas sobre los derechos, el apoyo y la protección de las víctimas de delitos, y por la que se sustituye la Decisión marco 2001/220/ JAI del Consejo. Versión electrónica: <http://www.boe.es/doue/2012/315/ L00057-00073.pdf>

FloRES, Glenn; Barton Laws; Sandra Mayo; Barry Zuckerman; Milagros Abreu; Leonardo Medina \& Eric Hardt. (2003) "Errors in medical interpretation and their potential clinical consequences in pediatric encounters." Pediatrics 111:1, pp. 6-14.

HALE, Sandra. (2007) Community Interpreting. Basingstoke: Palgrave Macmillan.

HALE, Sandra. (2011) Breaking through the language barrier: Empowering refugee and immigrant women to combat domestic and family violence. (Sin publicar. Informe consultado por cortesía de la autora.).

Huelgo, Vivian; Saveen Kaushat; Purvi Shah \& Catherine Shugrue dos Santos. (2006) "The Voice of Justice: Interpreting Domestic Violence Cases." Proteus. The Newsletter of The National Association of Judiciary Interpreters and Translators xv:2, pp 4-6.

JACOBS, Elizabeth A.; Niels Agger-Gupta; Alice Chen; Adam Piotrowski \& Eric J. Hardt. (2003) Language Barriers in Health Care Settings: An annotated bibliography of the research literature. Woodland Hills, California: The California Endowment. Versión electrónica: <http://www.hablamosjuntos.org/pdf_files/ Cal.Endow.Bibliography.pdf>

Kelly, Nataly \& Marjory Bancroft. (2007) "The Critical Role of Health Care Interpreting: Views from the Literature, Promising Practices and Lessons Learned in the United States." En: Epstein, Len (ed.) 2007. Culturally Appropriate Health Care by Culturally Competent Health Professionals. International Workshop Report. Caesarea, Israel: The Israel National Institute for Health Policy and Health Services Research, pp. 85-100.

Molina Gutiérrez, Macarena. (2006) "Los servicios de interpretación a disposición de las mujeres inmigrantes maltratadas y la nueva ley contra la violencia machista en España." Translation Journal 10:3. Versión electrónica: <http:// translationjournal.net/journal/37violencia.htm> 
ODA, Melanie \& Donna Joyette. (2003) "Interpreting for the perpetrator in the partner assault response program. The selection and training process." En: Brunette, Louise; Georges Bastin; Isabelle Hemlin \& Heather Clarke (eds.) 2003. The Critical Link 3: Interpreting in the Community: the Complexity of the Profession. Amsterdam \& Philadelphia: John Benjamins, pp. 147-161.

Ortega Herráez, Juan Miguel; Isabel Abril \& Anne Martin. (2009) "Community Interpreting in Spain: a comparative study of interpreters' self perception of role in different settings." En: Hale, Sandra; Uldis Ozolins \& Ludmila Stern (eds.) 2009. The Critical Link 5: Quality in Interpreting - A Shared Responsibility. Amsterdam \& Philadelphia: John Benjamins, pp. 149-167.

Ortega Herráez, Juan Miguel. (2010) Interpretar para la Justicia. Granada: Comares.

PÖCHHACKER, Frank \& Mira Kadric. (1999) "The Hospital Cleaner as Healthcare Interpreter: A Case Study.” The Translator 5:2, pp. 161-178.

PÖCHHACKER, Frank. (2000) "The Community Interpreter's Task: Self-Perception and Provider Views." En: Roberts, Roda; Silvana Carr; Diana Abraham \& Aideen Dufour (eds.) 2000. The Critical Link 2: Interpreters in the Community. Amsterdam \& Philadelphia: John Benjamins \& ATA, pp. 49-65.

PÖllabauer, Sonja. (2008) Summary report: Workpackage 3. MedInt: Development of a Curriculum for Medical Interpreters. Graz: University of Graz. Versión electrónica: <http://www.uni-graz.at/en/wp3_final_report_210508.pdf>

Polzin, Jennifer. (2007) "Interpreting in Domestic Violence Service Settings." The ATA Chronicle June, pp. 22-26.

RagA, Francisco \& Carmen Valero (eds.) (2006) Retos del Siglo XXI para la Lingüística Aplicada: Nuevo Mapa Lingüístico y Cultural de la Península Ibérica. Número monográfico de la Revista Española de Lingüística Aplicada (RESLA).

SÁNCHEZ-REYES, Sonsoles \& Manuel Martín Casado. (2005) "Intérpretes profesionales e intérpretes 'ad hoc' en un entorno sanitario." En: Valero, Carmen (ed.) 2005. Traducción como mediación entre lenguas y culturas. Alcalá de Henares: Universidad de Alcalá, pp. 139-146.

SCHWEDA-NiCHOLSON, Nancy. (1989) "Ad Hoc Court Interpreters in the United States: Equality, Inequality, Quality?” Meta 34:4, pp. 711-723.

TOledAno Buendía, Carmen. (2010) "Community Interpreting: breaking with 'the norm' through normalisation.” Jostrans 14. Versión electrónica: < http:// www.jostrans.org/archive.php? display=14>

Toledano Buendía, Carmen \& María Magdalena Fernández. (2012) "Las barreras lingüísticas en la atención a las víctimas extranjeras de violencia de género: un factor ignorado." En: Tacoronte Domínguez, María José; Anisa Azaovagh de la Rosa \& Amparo Romero Sánchez (eds.) 2012. Actas de las II Jornadas de Investigaciones Feministas y Análisis de Género. Avances y propuestas. Tenerife: Universidad de La Laguna. 
Toledano Buendía, Carmen \& Maribel del Pozo Triviño (eds.) (2014) La interpretación en contextos de violencia de género. Valencia: Tirant lo Blanch.

Unidad Orgánica de Violencia de GÉnero del Cabildo de Tenerife. (2011) Análisis del Cuestionario de evaluación de mediación lingüistica. Cabildo de Tenerife (inédito).

\section{NOTAS BIOGRÁFICAS / BIONOTES}

Carmen Toledano Buendía es Profesora Titular de Filología Inglesa y Alemana de la Universidad de La Laguna (ULL) y especialista en estudios descriptivos de traducción e interpretación. Cuenta con una larga experiencia formativa en interpretación de conferencias y TISP, ha impartido docencia de posgrado en centros nacionales y extranjeros. Asimismo, cuenta con amplia experiencia en el diseño, organización y gestión de formación especializada, habiendo coordinado el Máster en Interpretación de Conferencias y creado y dirigido el Experto Universitario en Traducción e Interpretación para los Servicios Comunitarios: mediadores lingüísticos (ULL). Forma parte del Grupo de investigación Fitispos, la Red Comunica (Observatorio Permanente sobre Interpretación en los Servicios Públicos en España) y el Instituto de la Mujer de la Universidad de La Laguna.

Carmen Toledano Buendía, Ph.D., is Senior Lecturer at the Department of English Philology at the University of La Laguna (ULL) (Tenerife) and specialist in descriptive translation and interpreting studies. She has extensive experience teaching conference and public service interpreting and in the design and management of specialized courses on interpreting. She is currently the academic director of the Experto Universitario en Traducción e Interpretación para los Servicios Comunitarios: mediadores lingüísticos and former academic coordinator of the Master en Interpretación de Conferencias (ULL). She is member of the Fitispos Research Group, Comunica Network (Observatorio Permanente sobre Interpretación en los Servicios Públicos en España) and the Instituto de la Mujer at the University of La Laguna.

María IsAbel Abril Martí es profesora de interpretación de la Universidad de Granada. Obtuvo su doctorado en interpretación en la misma universidad, en octubre de 2006, con una tesis sobre la definición y evolución de la Interpretación en los Servicios Públicos y la formación de intérpretes. Su actividad investigadora se centra en los campos de la interpretación y su didáctica (especialmente en la interpretación en los servicios públicos), en los que 
ha publicado diversos trabajos. Es responsable del Grupo de Investigación GRETI, La interpretación ante los retos de la mundialización: formación y profesión (HUM-737 de la Junta de Andalucía), y miembro de la Red Comunica. Asimismo, es coordinadora adjunta del Máster Oficial en Traducción e Interpretación (perfil investigador) de la Universidad de Granada.

MARÍA ISABEl ABRIL MARTí lectures in interpreting at the University of Granada. She holds a Ph.D. from the same University, with a dissertation on the definition and evolution of public service interpreting (PSI) and the training of interpreters, areas where she has focused her research to date. She is the head of the GRETI Research group (Group HUM-737: "Interpreting and the challenges of globalization - Training and the profession", funded by the Andalusian Regional Authorities), and a member of the Comunica Network. She is also assistant coordinator of the Máster Oficial en Traducción e Interpretación (perfil investigador) of the University of Granada.

MaRibel del Pozo TRIVIÑo es doctora en Traducción e Interpretación por la Universidad de Vigo y traductora e intérprete jurada inglés-español desde 1998. Ha desarrollado una intensa carrera profesional como traductora e intérprete y actualmente es profesora en la Universidad de Vigo. Tiene numerosas publicaciones relacionadas con la traducción jurídica y la traducción e interpretación en los servicios públicos y participa en varios proyectos de investigación relacionados con este ámbito. Forma parte del grupo de investigación GENTT (Universidad Jaume I), es miembro de la Asociación Ibérica de Estudiosos de la Traducción y la Interpretación (AIETI) y de la Red Comunica. Además, es miembro fundador de la Asociación Galega de Profesionais da Traducción e a Interpretación (AGPTI), miembro de la Asociación de Traductores e Intérpretes Jurados de Cataluña (ATIJC) y de la Asociación Profesional de Traductores e Intérpretes Judiciales y Jurados (APTIJ).

MARIBEl Del Pozo TRIVIÑo has a Ph.D. in Translation and Interpretation from the University of Vigo, Spain, where she is currently working as a lecturer. She was appointed as a sworn English-Spanish translator-interpreter in 1998 by the Ministry of Foreign Affairs. Her professional T\&I work experience is extensive and covers a wide range of fields. She has a great deal of publications on the different aspects of legal translation and Public Services T\&I, and is also currently involved in several research projects on the mentioned subjects. She is a member of the GENTT research group (University of Jaume I), the Iberian Association of T\&I Scholars (AIETI) and the Comunica Network. Besides the above, she is also a founding member of the Galician Professional 
T\&I Association (AGPTI), member of the Catalonian Sworn T\&I Association (ATIJC) and of the Professional Legal \& Sworn T\&I Association (APTIJ).

Laura Aguilera Ávila es profesora en el Departamento de Trabajo Social y Servicios Sociales de la Universidad de La Laguna. Con las titulaciones de Psicología y Trabajo Social, sus investigaciones se han centrado en el ámbito del género y de la violencia de género (Matud, Díaz, Aguilera, Rodríguez y Matud, 2003; Matud, Aguilera, Marrero, Moraza y Carballeira, 2003). También es de destacar su labor profesional, habiendo trabajado como psicóloga en un servicio municipal de atención a mujeres víctimas de violencia de género. Entre sus funciones destacan la evaluación, tratamiento individual y grupal, impulso de grupos de autoayuda, etc., formando parte de un equipo multidisciplinar.

Laura Aguilera Ávila lectures at the Social Work and Social Services Department at the University of La Laguna. She holds degrees in Psychology and Social Work, and has focused her research on the field of gender and battered women (Matud, Díaz, Aguilera, Rodríguez y Matud, 2003; Matud, Aguilera, Marrero, Moraza y Carballeira, 2003). She has extensive professional experience, and has worked as a psychologist in a municipal centre for women victims of gender-based violence. Her tasks include assessment, individual and group therapy, encouragement of self-help groups, etc. as part of a multidisciplinary team. 